\title{
Constrained selection
}

\author{
By G. M. TALLIS \\ Department of Statistics, The University of Adelaide, \\ South Australia
}

(Received September 25, 1984)

\section{INTRODUCTION}

Some years ago the author proposed an index which allowed progress to be made with respect to genetic economic value, while forcing certain characters to pre set optimum levels, see Tallis (1962). This procedure extended the work of Kempthorne and Nordskog (1959) who constructed a similar index which ensured that selection resulted in zero progress in some characters.

There has been a large amount written about constrained selection over recent years. Initially the trend was to extensions and generalisation of earlier results, Mallard (1972), but more recently the practical limitations to such indices have been a major worry. For this reason certain special cases have received particular attention; see for example Yamada et al. (1975) who consider selection progress with respect to specific characters as a main goal. The reader is referred to this paper for further details.

It is my purpose here to re-examine the 1962 set-up and to make certain adjustment. These will lead to a more complete discussion of the special cases and a fuller treatment of the whole minimisation process.

The plan of this paper is as follows. Firstly, the notation is established and the constraint problem specified. It is shown that the only way for the economic weights of constrained characters to enter the problem is by means of the constraints. This leads to the definition of economic distance and constraints are imposed in these terms.

Secondly, it is shown that minimising the variance of the difference between economic merit and the selection index with respect to the coefficients of the index, and subject to the constraints, is equivalent to maximising the correlation coefficient between genetic merit and the index under the same constraints. This point does not seem to be well known and it leads to easier calculations.

Subsequently two cases are examined. The situation where economic weights are zero for characters which are not subject to constraint is considered first. This leads directly to some of the special cases which have been reported in the literature. Finally, the situation of arbitrary economic weights for all characters is dealt with and a full, although complicated, solution to the general problem is achieved. 


\section{NOTATION}

With minor modifications, the notation of the earlier paper will be used and is listed here for convenience.

$C_{i} \quad$ character $i, i=1,2, \cdots, m$

$p_{i} \quad$ phenotype of $C_{i}$

$g_{i} \quad$ additive genotype of $C_{i}$

$a_{i}$ economic weight of $C_{i}$

$I \quad \sum_{1}^{m} b_{i} p_{i}=p^{\prime} b ;$ selection index

$P \quad V[p]$, the phenotypic covariance matrix

$G \quad V[g]$, the additive genetic covariance matrix

$\sigma_{I}{ }^{2} \quad V[I]$, the variance of $I$

$\Delta_{I}$ the selection differential of $I$

$\bar{g}_{i}$ the expected value of $g_{i}$ under.selection on $I$.

The mean of $C_{i}$ is $\mu_{i}$ and it is desired to move $\mu_{i}$ to $\mu_{i}+k_{i}$ for $i=1,2, \cdots, r$. The fate of the other $\mu_{j}, j=r+1, \cdots, m$, is of no concern.

To save space, put $g_{(1)}^{\prime}=\left(g_{1}, \cdots, g_{r}\right), g_{(2)}^{\prime}=\left(g_{r+1}, \cdots, g_{m}\right)$ with similar definitions for $a_{(1)}, a_{(2)}, \bar{g}_{(1)}$ and $\bar{g}_{(2)}$. Also, let $C\left[g_{(1)}, p\right]=G_{1}$, the $(r \times m)$ submatrix of $G$ consisting of the first $r$ rows; $C\left[g_{(2)}, p\right]=G_{2}$ is an $(m-r) \times m$ matrix and consists of the rest of $G$.

The problem treated in 1962 was: let $H=\alpha^{\prime} g$, then minimise

$$
Q(b)=V\left[H-p^{\prime} b\right]=V[H]-2 b^{\prime} G_{1}{ }^{\prime} a_{(1)}-2 b^{\prime} G_{2}{ }^{\prime} a_{(2)}+b^{\prime} P b
$$

with respect to $b$ subject to $C\left[g_{i} I\right]=k_{i}, i=1,2, \cdots, r$. The side conditions can be written as $G_{1} b=k$, and it is now quite clear that the solution will be independent of the vector of economic weights $a_{(1)}$ since it becomes absorbed by the lagrange multipliers when the constraint condition is introduced. Thus, set

$$
u(b)=Q(b)-2 b^{\prime} G_{1}{ }^{\prime} \lambda^{*}=V[H]-2 b^{\prime} G_{2}{ }^{\prime} a_{(2)}+b^{\prime} P b-2 b^{\prime} G_{1}{ }^{\prime} \lambda
$$

where $\lambda=\lambda^{*}+a_{(1)}$, and $a_{(1)}$ cannot contribute to the solution.

Therefore, the only way for the economic weights, $a_{i}, i=1,2, \cdots, r$ to enter the problem is by means of the constraints. If the $a_{i}$ are known, then it seems sensible to define the "economic distances" $d_{i}=a_{i} k_{i}$. Now, if these distances are to be achieved simultaneously in a certain number of applications of $I$, we must have

$$
\bar{g}_{(1)}=\frac{G_{1} b i}{\sigma_{I}}=\alpha d
$$

for some $\alpha$ yet to be determined and $i$ the standardised selection differential. Thus, $G_{1} b=\theta d$, again where $\theta$ is to be determined.

Putting $H=1^{\prime} g_{(1)}+a_{(2)}^{\prime} g_{(2)}, 1$ being a vector of $r 1^{\prime}$ s, then 


$$
H=\frac{C[H, I]}{\sigma_{I}} i=1^{\prime} \bar{g}_{1}+a_{(2)}^{\prime} \bar{g}_{(2)}=\frac{\left(1^{\prime} G_{1}+a_{(2)}^{\prime} G_{2}\right) b i}{\sigma_{I}} .
$$

Moreover, the vector $b\left(\theta^{*}\right)$ which satisfies $\inf _{\theta} \inf _{G_{1} b=\theta d} V\left[H-p^{\prime} b\right]$ also satisfies

$$
\sup _{\theta} \sup _{G_{1} b=\theta d} \frac{C[H, I] i}{\sigma_{I}}=\sup _{\theta} \sup _{G_{1} b=\theta d}\left[\frac{\theta 1^{\prime} d i+a_{(2)}^{\prime} G_{2} b i}{\sigma_{I}}\right] .
$$

To show this let $B=\left\{b ; G_{1} b=\theta d, \theta\right.$ arbitrary $\}$ and suppose $\inf _{b \in B} V\left[H-p^{\prime} b\right]$ occurs at $b_{0}$.

Now for any other $b_{1} \in B$ define $b_{2}=\theta_{1} b_{1}, \theta_{1}{ }^{2}=b_{0}{ }^{\prime} P b_{0} /\left(b_{1}{ }^{\prime} P b_{1}\right)$. Then certainly $b_{0}{ }^{\prime} P b_{0}=b_{2}{ }^{\prime} P b_{2}$ and $\rho\left[H, p^{\prime} b_{2}\right]=\rho\left[H, p^{\prime} b_{1}\right]$. But, by choice of $b_{0}, V\left[H-p^{\prime} b_{0}\right] \leq$ $V\left[H-p^{\prime} b_{2}\right]$ and remembering that

$$
V\left[H-p^{\prime} b\right]=V[H]+b^{\prime} P b-2 \rho\left[H, p^{\prime} b\right]\left(b^{\prime} P b\right)^{1 / 2}(V[H])^{1 / 2}
$$

it follows that

$$
\rho\left[H, p^{\prime} b_{0}\right] \geq \rho\left[H, p^{\prime} b_{2}\right]=\rho\left[H, p^{\prime} b_{1}\right]
$$

3. THE CASE $a_{(2)}=0$

Because most practical interest will centre on the case $a_{(2)}=0$, this will be dealt with first. In this form,

and

$$
u(b)=V[H]+b^{\prime} P b-2 b^{\prime} G_{1}{ }^{\prime} \lambda
$$

$$
\begin{gathered}
\frac{1}{2} \frac{\partial u}{\partial b}=P b-G_{1}{ }^{\prime} \lambda=0, \\
b=P^{-1} G_{1}{ }^{\prime} \lambda ; G_{1} b=\theta d=G_{1} P^{-1} G_{1}{ }^{\prime} \lambda ; \quad \lambda=\left(G_{1} P^{-1} G_{1}\right)^{-1} \theta d
\end{gathered}
$$

so that $b(\theta)=\theta P^{-1} G_{1}{ }^{\prime}\left(G_{1} P^{-1} G_{1}{ }^{\prime}\right)^{-1} d$.

Now $H=i \theta 1^{\prime} d / \sigma_{I}(\theta)$, where ${\sigma_{I}}^{2}(\theta)=b^{\prime}(\theta) P b(\theta)=\theta^{2} d^{\prime}\left(G_{1} P^{-1} G_{1}^{\prime}\right)^{-1} d=\theta^{2}{\sigma_{I}}^{2},\left(\sigma_{I}{ }^{2}(1)\right.$ $\left.=\sigma_{I}^{2}\right)$, so that

$$
H=i 1^{\prime} d / \sigma_{I}
$$

and is independent of $\theta$. The choice of $\theta=1$ is therefore convenient and appropriate.

Incidentally, in this instance it is exceedingly easy to see that $H$ is a maximum since

$$
\sup _{G_{1} b=\theta d} \frac{1^{\prime} G_{1} b i}{\sigma_{I}}=\frac{\theta 1^{\prime} d i}{\inf _{G_{1} b=\theta d} \sigma^{I}}=\frac{1^{\prime} d i}{\sqrt{d^{\prime}\left(G_{1} P^{-1} G_{1}^{\prime}\right)^{-1} d}}
$$

When $a_{i}=1, i=1,2, \cdots, r$, the vector $a$ becomes

and

$$
b=P^{-1} G_{1}^{\prime}\left(G_{1} P^{-1} G_{1}\right)^{-1} k
$$




$$
H=i 1^{\prime} k / \sqrt{k^{\prime}\left(G_{1} P^{-1} G_{1}^{\prime}\right)^{-1} k}
$$

which is the same as the result discussed by Yamada et al. (1975). Assigning $a_{i}=1$ may occur when the actual economic weights are not known and it is elected to simply select towards an optimum, irrespective of economics.

The case $a_{i}=1, i=1,2, \cdots, r$, and $r=m$ corresponds to the case considered by Tallis (1962), (1968), by Rohde and Tallis (1969) and essentially, by Pesek and Baker (1969).

\section{THE CASE $a_{(2)} \neq 0$}

When $a_{(2)} \neq 0, u(b)=V[H]-2 b^{\prime} G_{2}{ }^{\prime} a_{(2)}+b^{\prime} P b-2 b^{\prime} G_{1}{ }^{\prime} \lambda$ and

$$
\begin{gathered}
\frac{1}{2} \frac{\partial u}{\partial b}=P b-G_{2}{ }^{\prime} a_{(2)}-G_{1}{ }^{\prime} \lambda=0 \\
b=P^{-1}\left(G_{2}{ }^{\prime} a_{(2)}+G_{1}{ }^{\prime} \lambda\right) ; G_{1} b=\theta d=G_{1} P^{-1}\left(G_{2}{ }^{\prime} a_{(2)}+G_{1}{ }^{\prime} \lambda\right) ; \\
\lambda=\left(G_{1} P^{-1} G_{1}\right)^{-1}\left(\theta d-G_{1} P^{-1} G_{2}{ }^{\prime} a_{(2)}\right)
\end{gathered}
$$

so that

$$
b(\theta)=\left[I-P^{-1} G_{1}{ }^{\prime}\left(G_{1} P^{-1} G_{1}\right)^{-1} G_{1}\right] P^{-1} G_{2}{ }^{\prime} a_{(2)}+P^{-1} G_{1}{ }^{\prime}\left(G_{1} P^{-1} G_{1}\right)^{-1} \theta d .
$$

Now substituting $b(\theta)$ into $V\left[H-p^{\prime} b(\theta)\right]$, the resulting expression is a quadratic in $\theta$ with a minimum at

$$
\theta^{*}=a_{(2)}^{\prime} G_{2} P^{-1} G_{1}{ }^{\prime}\left(G_{1} P^{-1} G_{1}{ }^{\prime}\right)^{-1} d /\left[d^{\prime}\left(G_{1} P^{-1} G_{1}{ }^{\prime}\right)^{-1} d\right]
$$

giving $b\left(\theta^{*}\right)$ as the optimum set of coefficients to use for the index.

This result is explicit, complicated and not very pleasing. Nevertheless, it does round out the technical exercise and caters for the criticism that the original index was not optimal, Mallard (1972). It is equivalent to $b\left(\alpha_{m}\right)$ of Harville (1975), who obtained this result with somewhat more effort by maximising the correlation between $H$ and $I$.

For further practical discussion and a numerical example see Yamada et al. (1975).

Part of the above derivation has been adapted from Tallis and Chesson (1975).

The author wishes to thank Dr. R. J. Baker for enlightening communications leading to the writing of this note and to the referee for valuable suggestions.

\section{REFERENCES}

HARVILle, D. A. (1975) Index selection with proportional constraints. Biometric 31, 223-225. Kempthorne, O. and NoRDskoG, A. W. (1959) Restricted selection indices. Biometric 15, 10-19. MALlARD, J. (1972) La theorie et le calcul des index de selection avec restrictions: synthese critique. Biometric 28, 713-735.

Pesek, J. and BAker, R. J. (1969) Desired improvement in relation to selection indices. Can. J. Plant Sci. 49, 803-804. 
RoHDE, C. H. and TALLIS, G. M. (1969) Exact first- and second-order moments of estimates of components of covariance. Biometrika 56, 517-525.

TAllis, G. M. (1962) A selection index for optimum genotype. Biometrics 18, 120-122.

TALLIS, G. M. (1968) Selection for an optimum growth curve. Biometris 24, 169-177.

TAllis, G. M. and Chesson, P. L. (1975) Two optimisation problems of constrained selection theory. Technical Paper No. 5, Department of Statistics, The University of Adelaide.

Yamada, Y., Yokouchi, K. and Hishida, A. (1975) Selection index when genetic gains of individual traits are of primary concern. Jpn. J. Genetics 50, 33-41. 